INTERNATIONAL ASTRONOMICAL UNION

SYMPOSIUM NO. 220

\title{
DARK MATTER IN GALAXIES
}

Edited by: S. D. RYDER, D. J. PISANO, M. A. WALKER and K. C. FREEMAN

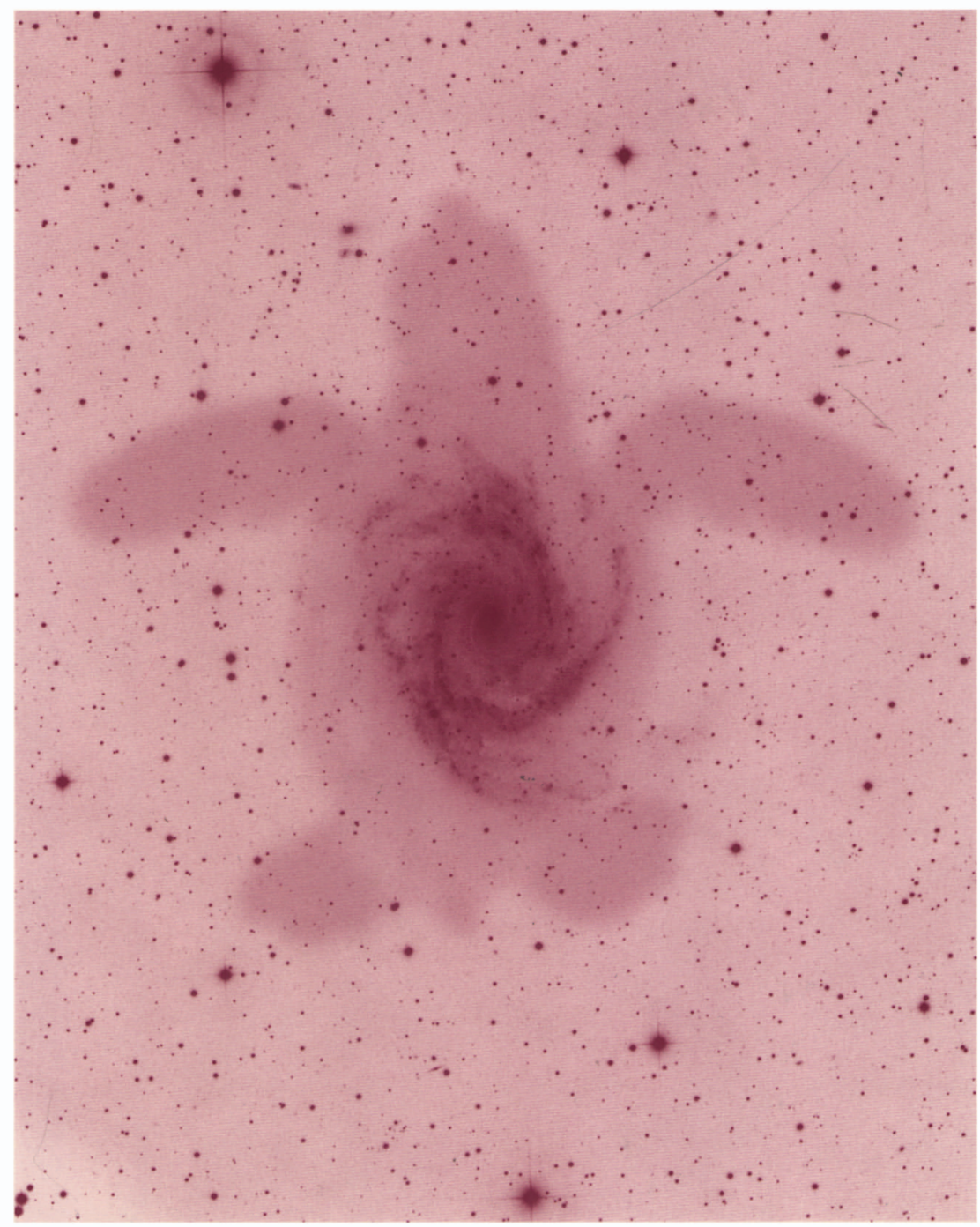

\section{ॠA}

INTERNATIONAL ASTRONOMICAL UNION

PUBLISHER:

ASTRONOMICAL SOCIETY OF THE PACIFIC 
DARK MATTER IN GALAXIES

IAU SYMPOSIUM VOLUME 220

Cover Illustration:

Courtesy of David Malin (http://www.davidmalin.com) 
ASTRONOMICAL SOCIETY OF THE PACIFIC 390 Ashton Avenue - San Francisco - California - USA 94112-1722

Phone: (415) 337-1100 E-Mail: service@astrosociety.org

Fax: (415) 337-5205 Web Site: www.astrosociety.org

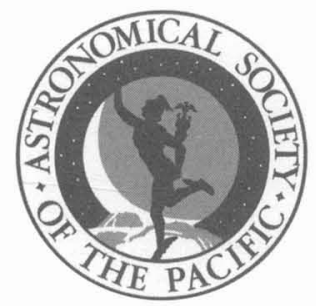

\title{
ASP-CS VOLUMES \& IAU PUBLICATIONS - EDITORIAL STAFF
}

\author{
Managing Editor: D. H. McNamara \\ Associate Managing Editor: J. W. Moody \\ Production Manager: Enid L. Livingston
}

PO Box 24463, Room 211 - KMB, Brigham Young University, Provo, Utah, 84602-4463

Phone: (801) 422-2111 Fax: (801) 422-0624 E-Mail: pasp@byu.edu

LaTeX-Computer Consultant: T. J. Mahoney (Spain)-tjm@11.iac.es

A listing of other volumes published by the

Astronomical Society of the Pacific, is cited at the back of this volume 


\section{INTERNATIONAL ASTRONOMICAL UNION \\ 98bis, Bd Arago - F-75014 Paris - France \\ Tel: +33143258358 E-mail: iau@iap.fr \\ Fax: +33 143252616 Web Site: www.iau.org}

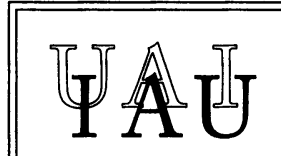

\section{DARK MATTER IN GALAXIES}

Proceedings of the 220th Symposium of the International Astronomical Union held during the IAU General Assembly XXV

Sydney, Australia

21-25 July 2003

Edited by

S. D. RYDER

Anglo-Australian Observatory, PO Box 296, Epping, NSW 1710, Australia

D. J. PISANO

Australia Telescope National Facility, PO Box 76

Epping, NSW 1710, Australia

M. A. WALKER

School of Physics, University of Sydney A28, Sydney NSW 2006, Australia

and

K. C. FREEMAN

Research School of Astronomy \& Astrophysics, Mt Stromlo Observatory

Cotter Road, Weston Creek, ACT 2611, Australia 
(C) 2004 by International Astronomical Union All Rights Reserved

No part of the material protected by this copyright notice may be reproduced or utilized in any form or by any means - graphic, electronic, or mechanical including photocopying, taping, recording or by any information storage and retrieval system, without written permission from the IAU.

Library of Congress Cataloging in Publication Data

Main entry under title

LOC \#: $\quad 2004105812$

ISBN: $\quad 1-58381-167-2$

IAU Publications - First Edition

Published on behalf of the IAU by: Astronomical Society of the Pacific

Printed in United States of America by Sheridan Books, Ann Arbor, Michigan 


\section{Contents}

Preface $\ldots \ldots \ldots \ldots \ldots \ldots \ldots \ldots \ldots \ldots \ldots \ldots \ldots \ldots \ldots \ldots$

\section{Part 1. Conference Summary}

Conference Summary . . . . . . . . . . . . . . . . 3 J. Binney

Part 2. Introduction to Dark Matter in Galaxies

Alternatives to Dark Matter (?) . . . . . . . . . . . . . 17

A. Aguirre

What is the Evidence for Dark Matter? . . . . . . . . . . . . . 27

J. A. Sellwood

Dark Matter in Galaxies: Observational overview . . . . . . . . . . .

A. Bosma

\section{Part 3. Central Density Cusps, Thin Disks, and Dark Halo}

\section{Substructure}

Summary talk: How serious are the problems faced by CDM: cusps, thin

disks, and halo substructure . . . . . . . . . . . . .

J. R. Primack

The Inner Density Cusp of Cold Dark Matter Halos $\ldots \ldots \ldots$. . . . 61

J. F. Navarro

CDM in LSB Galaxies: Toward the Optimal Halo Profile . . . . . . . . 69

W. J. G. de Blok

The Kinematics in the Cores of Low Surface Brightness Galaxies . . . .

R. A. Swaters, M. A. W. Verheijen, M. A. Bershady and D. R. Andersen

Lensing Diagnostics of Halo Substructure $\ldots \ldots \ldots \ldots$ S. Mao

Small-scale Substructure in Dark Matter Haloes: Where Does Galaxy Formation Come to an End? . . . . . . . . . . . . . . . . . . J. E. Taylor, J. Silk and A. Babul

Inner Structure of Dark Matter Halos

T. Fukushige, A. Kawai and J. Makino 


\section{Part 4. Lensing}

The dark matter content of lensing galaxies at $1.5 R_{e} \ldots \ldots$. . . . . 103

P. L. Schechter and J. Wambsganss

Determining the Properties of Galaxy 2237+0305 using Gravitational

Lensing . . . . . . . . . . . . . . . . .

C. Trott and R. Webster

Decomposition of the Visible and Dark Matter Mass Profiles in the

Einstein Ring $0047-2808$. . . . . . . . . . . . . . . . . . . .

$S$. Dye and S. Warren

Evidence for Halo Microlensing from a Survey of M31 . . . . . . . . . .

A. P. S. Crotts, R. R. Uglesich, E. A. Baltz, J. de Jong, R. P. Boyle and C. J. Corbally

MEGA: Microlensing Exploration of the Galaxy and Andromeda . . . .

D. R. Alves, E. A. Baltz, A. P. S. Crotts, A. Bergier, P. Cseresnjes and

A. Gersch

Lighting up the dark and dim in the Andromeda Galaxy . . . . . . . . . E. Kerins

The EROS2 Microlensing Study of the Galaxy . . . . . . . . . . . . 131

C. Hamadache

On Planetary-Mass Compact Objects as Dark Matter . . . . . . . . .

E. Zackrisson, C. Persson and N. Bergvall

Astrometric study of MACHO halo distribution in our Galaxy . . . . .

K. Ohnishi, M. Hosokawa and T. Fukushima

Astrometric Microlensing by Finite-size Lenses . . . . . . . . . . . . R. Takahashi

Direct Mapping of Massive Compact Objects in Extragalactic Dark Halos K. T. Inoue and M. Chiba

Quasar Mesolensing as a Probe of CDM Substructures . . . . . . . . . . 141 A. Yonehara, M. Umemura and H. Susa

Gravitational Lens Statistics as a Probe of Halo Profiles . . . . . . . . . 143 M. Oguri

Mass-to-Light Ratios of Early- and Late-Type Galaxies . . . . . . . . . T. G. Brainerd and M. A. Specian

\section{Part 5. Clusters and Ellipticals}

X-Ray Constraints on Dark Matter in Galaxy Clusters and Elliptical Galaxies: A View from Chandra and XMM . . . . . . . . . . . . D. A. Buote

The dark matter halos of spheroidal galaxies and clusters of galaxies . . T. Treu, L. V. E. Koopmans, D. J. Sand, G. P. Smith and R. S. Ellis

Elliptical Galaxies: Darkly Cloaked or Scantily Clad? . . . . . . . . . .

A. J. Romanowsky, N. G. Douglas, K. Kuijken, M. R. Merrifield,

M. Arnaboldi, N. R. Napolitano, H. Merrett, M. Capaccioli,

K. C. Freeman and O. Gerhard 
Early-type Galaxy Halo Dynamics inferred using the PN Spectrograph .

N. G. Douglas, A. J. Romanowsky, K. Kuijken, M. R. Merrifield,

N. R. Napolitano, M. Arnaboldi, K. C. Freeman, M. Capaccioli and

O. Gerhard

Is there a dichotomy in the Dark Matter as well as in the Baryonic Matter properties of ellipticals?

N. R. Napolitano, M. Capaccioli, 'M. Arnaboldi, M. R. Merrifield,

N. G. Douglas, K. Kuijken, A. J. Romanowsky and K. C. Freeman

The Halo Mass Distribution of Field and Cluster Early-Type Galaxies . M. P. Bergmann, A. Forestell, K. Gebhardt and I. Jørgensen

The dark halo in the elliptical galaxy NGC 3108 . . . . . . . . . . . . . G. I. G. Józsa, T. A. Oosterloo, R. Morganti and D. Vergani

Orbital structure of triaxial galaxies . . . . . . . . . . . . . .

G. van de Ven, E. Verolme, M. Cappellari, P. T. de Zeeuw

ERO R1 in the field of CL0939+4713 - Evidence for an S0-like galaxy at $z \sim 1.5 \ldots \ldots \ldots \ldots \ldots \ldots$

M. Iye, N. Kashikawa, M. Imanishi, T. Kodama, M. Chiba,

K. Shimasaku, S. Okamura, S. Miyazaki, C. Simpson, N. Kobayashi,

H. Terada, M. Goto and F. Iwamuro

$1.2 \mathrm{~mm}$ emission from Cen $\mathrm{A} \ldots \ldots \ldots \ldots$

Z. Banhidi, R. Chini and M. Albrecht

Directions of cosmic anisotropies . . . . . . . . . . . . . .

K. Bajan, P. Flin and V. N. Pervushin

\section{Part 6. The Galaxy}

Dark Matter Constraints from the Sagittarius Dwarf and Tail System .

S. R. Majewski, D. R. Law, K. V. Johnston, M. F. Skrutskie and

M. D. Weinberg

The Mass of the Galaxy from Large Samples of Field Horizontal-Branch

Stars in the SDSS Early Data Release . . . . . . . . . . . . . . . . .

T. C. Beers, M. Chiba, T. Sakamoto, R. Wilhelm, C. Allende Prieto,

J. Sommer-Larsen, H. J. Newberg, B. Yanny, B. Marsteller and J. R. Pier

Galactic Disk Surface Density in the Solar Neighbourhood .

W. F. van Altena, V. I. Korchagin, T. M. Girard, D. I. Dinescu and

T. V. Borkova

Search for cool white dwarfs with GSC2 . . . . . . . . . . . . .

D. Carollo, A. Spagna, M. G. Lattanzi, R. L. Smart, S. T. Hodgkin,

L. Terranegra and B. McLean

Mapping the Remote Milky Way Halo using BHB stars at $70<r<130 \mathrm{kpc}$

L. Clewley, S. J. Warren, P. Hewett, M. Wilkinson and N. W. Evans

Rotation curve of our Galaxy and field galaxies . . . . . . . . . .

D. Russeil, O. Garrido, P. Amram and M. Marcelin

Mass of the Milky Way ....................

O. I. Wong, M. J. Drinkwater, J. B. Jones, M. D. Gregg and

K. C. Freeman 
Using Globular Clusters to Test Newton's Law of Gravity . . . . . . . .

R. Scarpa, G. Marconi and R. Gilmozzi

\section{Part 7. Baryonic Dark Matter}

Galaxy Formation and Baryonic Dark Matter . . . . . . . . . . . . 219

F. Combes

Cosmic Matter Distribution: Cosmic Baryon Budget Revisited . . . . . 227 M. Fukugita

The visible matter - dark matter coupling . . . . . . . . . . . . 233

R. Sancisi

Cold Molecular Gas as Baryonic Dark Matter . . . . . . . . . . .

D. Pfenniger

Cold Molecular Gas as a Possible Component of Dark Matter in the Outer

Parts of Disk Galaxies ... . . . . . . . . . . . . . . .

R. J. Allen and R. Diaz-Miller

Molecular hydrogen as dark mass in dwarf galaxies . . . . . . . . .

P. R. Williams, C. Marzok, S. Myers and A. H. Nelson

\section{Part 8. Bars}

Bars and the connection between dark and visible matter . . . . . . .

E. Athanassoula

The dark matter density problem in massive disk galaxies . . . . . . .

B. J. Weiner

Fast bars in SB0 galaxies

E. M. Corsini, J. A. L. Aguerri and V. P. Debattista

Boxy isophotes in face-on views of barred galaxies

P. A. Patsis, C. Skokos and E. Athanassoula

Formation of inner rings in 3D potentials of barred galaxies

P. A. Patsis, C. Skokos and E. Athanassoula

The Dark Matter Content of Barred Spiral Galaxies . . . . . . . . . .

G. Petitpas, M. Das, P. Teuben and S. Vogel

\section{Part 9. Disks}

Properties of Dark Matter Halos in Disk Galaxies . . . . . . . . . . . .

R. S. de Jong, S. Kassin, E. F. Bell and S. Courteau

The mass distribution in early type disk galaxies . . . . . . . . . . .

E. Noordermeer, T. van der Hulst, R. Sancisi and R. Swaters

Rotating Halos and Heavy Disks: The Case of NGC 2915 . . . . . . .

F. S. Masset and M. Bureau

Evolution of Galaxies in Triaxial Halos with Figure Rotation . . . . . . K. Bekki and K. C. Freeman

SAURON dynamical modeling of NGC 2974 . . . . . . . . . . . . . . .

D. Krajnović, M. Cappellari, E. Emsellem, R. McDermid and

P. T. de Zeeuw 
Radial Distributions of Dark and Luminous Matter in Bright Spiral

Galaxies . . . . . . . . . . . . . . . . .

S. A. Kassin, R. S. de Jong and R. W. Pogge

The stellar Mass-to-Light ratio in disc galaxies . . . . . . . . . . . .

L. Portinari, J. Sommer-Larsen and R. Tantalo

Cores vs. Cusps: Dark Matter Density Profiles in Spirals . . . . . . . .

G. Gentile, U. Klein, P. Salucci and D. Vergani

Amplitude of Spiral Arms and Dark Matter . . . . . . . . . . .

P. Grosbøl

Nuclear Spirals and Supermassive Black Holes

H. B. Ann and P. Thakur

Tracing the relation between black holes and dark haloes

P. Buyle, M. Baes and H. Dejonghe

High-resolution CO Survey of Virgo Spirals at Nobeyama - Massive Cores inferred from Central Rotation Curves . . . . . . . . . . . . . . . . Y. Sofue, H. Nakanishi, S. Onodera, J. Koda and K. Kohno

Mass Determination in the Late-Type Spiral NGC 5457

I. Puerari, M. Valdez-Gutiérrez and M. Rosado

The Opacity of Spiral Galaxies from Counts of Distant Background

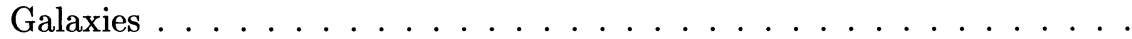

B. W. Holwerda, R. A. González, R. J. Allen and P. C. van der Kruit

Bulge Formation in Late-type Galaxies - Cuspy- vs Soft-core Dark

Matter Density Profiles . . . . . . . . . . . . . . . .

J.-H. Huang, Z.-G. Deng and Y.-N. Fu

Dark Matter Distribution in Nearby Galaxies

O. Garrido, P. Amram, C. Carignan, S. Blais-Ouellette, M. Marcelin and

D. Russeil

Dynamical Modeling using Evolutionary Algorithms . . . . . . . . . . A. Mathieu

Part 10. Low Surface Brightness Galaxies

A 3D Optical Spectroscopy Study of Low Surface Brightness Galaxies .

L. Chemin, P. Amram, C. Carignan, C. Balkowski, W. van Driel,

V. Cayatte, O. Hernandez, J. Boulesteix and M. Marcelin

The Central Dynamics of Blue Low Surface Brightness Galaxies . . . .

E. Zackrisson and N. Bergvall

Central DM density cuspiness in LSB's: a stellar kinematics approach .

A. Pizzella, E. M. Corsini, F. Bertola, L. Coccato, J. Magorrian, M. Sarzi and J. G. Funes

The $\sigma_{c}-V_{\text {circ }}$ correlation in high and low surface brightness galaxies . . A. Pizzella, E. Dalla Bontà, E. M. Corsini, L. Coccato and F. Bertola

A search for LSB dwarf galaxies in various environments . . . . . . . . . S. Roberts, J. Davies and S. Sabatini

Dust and the observed dark matter content of galaxies . . . . . . . . .

M. Baes, H. Dejonghe and J. I. Davies 
Part 11. Dwarf Galaxies

Kinematics of Extremely Faint Dwarf Galaxies . . . . . . . . . . . . . . 347

A. Begum and J. N. Chengalur

Dark Matter in Dwarf Galaxies: High Resolution Observations . . . . .

A. D. Bolatto, J. D. Simon, A. Leroy and L. Blitz

Extreme Dark Matter Dominated Dwarfs . . . . . . . . . . . . . .

M. I. Wilkinson, J. T. Kleyna, N. W. Evans and G. F. Gilmore

Evidence for Light-weight Local Group Dwarf Spheroidal Galaxies . . . 365 J. R. Kuhn and D. Kocevski

A Radial Velocity Dispersion Profile for the Fornax Dwarf Spheroidal Galaxy

M. G. Walker, M. Mateo, E. W. Olszewski, M. Woodroofe, X. Wang and J. Joyce

H I Rotation of Dwarf Galaxies with Unusually High H I Mass-to-Light Ratios ........................

B. E. Warren, H. Jerjen and B. S. Koribalski

Inner Halo Shapes of Dwarf Galaxies: Resolving the Cusp/Core Problem K. Spekkens and R. Giovanelli

A high-resolution rotation curve of NGC 6822 . . . . . . . . . . . . .

W. J. G, de Blok, D. T. F. Weldrake and F. Walter

\section{Part 12. Dark and Visible Matter Scaling Relations}

Scaling Laws for Dark Matter Halos in Late-Type and Dwarf Spheroidal Galaxies . . . . . . . . . . . . . . . . . . J. Kormendy and K. C. Freeman

The Relationship between Stellar and Halo Masses of Disk Galaxies at $z=0.2-1.2 \ldots \ldots \ldots \ldots \ldots \ldots$

C. J. Conselice, K. Bundy, R. S. Ellis, J. Brinchmann and N. Vogt

Polar Ring Galaxies and the Tully-Fisher relation: implications for the dark halo shape . . . . . . . . . . . . . . . . . . . M. Arnaboldi, E. Iodice, F. Bournaud, F. Combes, L. S. Sparke, W. van Driel and M. Capaccioli

Tully-Fisher Relations from an HI-Selected Sample . . . . . . . . . . .

M. J. Meyer, M. A. Zwaan, R. L. Webster and S. E. Schneider

The Tully-Fisher Relation for Hickson Compact Groups . . . . . . . . .

P. Amram, C. Mendes de Oliveira, H. Plana and C. Balkowski

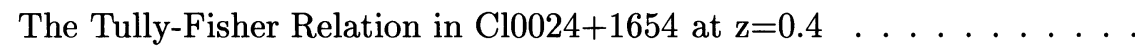
A. J. Metevier and D. C. Koo

Galaxy Scaling Relations as a Result of Secular Evolution . . . . . . . . $X$. Zhang 


\section{Part 13. The Shapes and Extents of Dark Halos}

The shapes of simulated dark matter halos

V. Springel, S. D. M. White and L. Hernquist

The Galactic Halo and CDM $\ldots \ldots \ldots \ldots \ldots \ldots$

M. R. Merrifield

Properties of galaxy dark matter halos from weak lensing . . . . . . 439

H. Hoekstra, H. K. C. Yee and M. D. Gladders

Observational Constraints on the Physical Parameters of Dark Matter

Halos . . . . . . . . . . . . . . . . . . . . . . . . . . 447

C. Carignan

What X-rays tell us about dark matter halos $\ldots \ldots \ldots \ldots \ldots . \ldots 455$ Y. P. Jing

Measuring Dark Matter Halos by Modeling Interacting Galaxies . . . 461 C. Theis

Orbit Evolution of Satellite Galaxies in Dark Matter Haloes . . . . . . . 463

A. Just

\section{Part 14. Angular Momentum}

The Origin and Distribution of Angular Momentum in Galaxies . . . . 467 J. R. Primack

Angular Momentum in Groups from Cosmological Simulations . . . . 477 $J$. Bailin and M. Steinmetz

On the angular momenta of galaxy structures . . . . . . . . . . . . 479 P. Flin, W. Godłowski and M. Szydłowski

\section{Part 15. Direct Detection of Elementary Particles}

WIMP direct detection and halo structure . . . . . . . . . . . . 483

A. M. Green

Accurate (In)Direct Detection Rates for Neutralinos . . . . . . . . . . 489

M. Schelke, J. Edsjö and P. Ullio

Study on Neutron-induced Background in the CRESST Experiment . . 491

H. Wulandari, F. von Feilitzsch, M. Huber, T. Jagemann, J. Jochum,

T. Lachenmaier, J.-C. Lanfranchi, W. Potzel, W. Rau, M. Stark and

S. Waller

CRESST Detectors for Nonbaryonic Cold Dark Matter Particles . ...

T. Jagemann

Dark Matter Search Experiments At Boulby Mine . . . . . . . . . . 495

S. M. Paling

Can quantum theory explain dark matter? . . . . . . . . . . . 497

A. D. Ernest

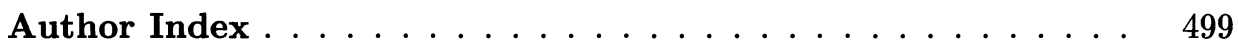

